\title{
Characterization of tribological protective films and friction wear by IR radiometry of thermal waves
}

\author{
by G. Kalus ${ }^{1}$, B.K. Bein ${ }^{1}$, J. Pelzl ${ }^{1}$, H. Bosse ${ }^{2}$, and A. Linnenbrügger ${ }^{3}$
}

${ }^{1}$ Exp. - Phys. III, AG Festkorperspektroskopie, Ruhr-Universitat, D-44780 Bochum, FR Germany;

${ }^{2}$ Institut für Maschinenkonstruktion, Technische Universitat Otto von Guericke, D-39016 Magdeburg, FR Germany; ${ }^{3}$ Institut für Reibungstechnik u. Maschinenkinetik, Technische Universitat Clausthal, D-38678 Clausthal-Zellerfeld, FR Germany

\begin{abstract}
Photothermal depth profiling based on IR detection of thermal waves has been applied to tribological films, before and after exposure to friction wear. It has been found that it is mainly the thermal conductivity of the protective film, which is reduced owing to friction wear and induced stresses.
\end{abstract}

\section{Amorphous films in tribological systems}

Thin amorphous films, deposited on metal substrates by plasma deposition or chemical vapour deposition are used in tribological systems to reduce the effects of friction wear [1] or to guarantee appropriate heat transfer conditions at the transition between the fluid lubricant and the solid contact [2].

Here, photothermal depth profiling based on IR detection of thermal waves [3] is applied to analyze such coatings or films, mainly consisting of diamond-like carbon or diamond-like carbon with some metallic additives. The thickness of the measured coatings varies between 2.5 and $4 \mu \mathrm{m}$. Some of the analyzed samples have got metallic bond layers between coating and substrate. Additionally, a virgin sample and samples after exposure to friction wear are compared, and the changes of the thermal depth profiles owing to the exposure to friction are interpreted quantitatively.

Subsequently, in section 2 the experimental device and method are briefly reviewed. In section 3 the experimental results are presented and interpreted quantitatively.

\section{Depth profiling based on IR detection of thermal waves}

In these depth profiling studies, the thermal waves have been excited by using an $\mathrm{Ar}^{+}$ laser beam as heating source with an effective beam power of $600 \mathrm{~mW}$ at the heated spot of a diameter of about $2.5 \mathrm{~mm}$. The laser beam has been intensity-modulated with the help of an electro-optical modulator. To resolve the comparatively thin layers, the modulation frequency has been varied up to $80 \mathrm{kHz}$. For the radiometric detection of the thermal response a photoconductive $\mathrm{HgCdTe}$ detector and an IR optical system consisting of a $\mathrm{CaF}_{2}$ lens and a $\mathrm{Si}$ cut-on filter just infront of the detector have been used, which allow a detectable wavelength interval of $1 \mu \mathrm{m}<\lambda<9 \mu \mathrm{m}$. The diameter of the $\mathrm{CaF}_{2}$ lens is $100 \mathrm{~mm}$ and its focal length $f_{L}=100 \mathrm{~mm}$, giving a maximum solid angle of $\Omega=0.196$ steradians for the radiant flux collected in a $2 \mathrm{f}_{\mathrm{L}}$ image. A two-phase lock-in amplifier is used to filter the comparatively small thermal wave response from the high radiation background and to analyze the thermal wave with respect to its amplitude and phase lag relative to the modulated laser beam. The measurements are run under computer control and the measured data are registered as functions of the modulation frequency. A schematic of the measuring system is shown in figure 1.

The radiometric signal, measured for the IR emission of a solid at stationary temperature 
$T$, can be described by

$$
M(T)=C \varepsilon_{e f f}\left(T, \lambda_{1}, \lambda_{2}\right) \int_{\lambda_{1}}^{\lambda_{2}} F(\lambda) R^{*}(\lambda) W_{\lambda}(\lambda, T) \mathrm{d} \lambda
$$

where the effective emissivity is given by

$$
\varepsilon_{\text {eff }}\left(T, \lambda_{1}, \lambda_{2}\right)=\int_{\lambda_{1}}^{\lambda_{2}} \varepsilon(\lambda, T) F(\lambda) R^{*}(\lambda) W_{\lambda}(\lambda, T) \mathrm{d} \lambda / \int_{\lambda_{1}}^{\lambda_{2}} F(\lambda) R^{*}(\lambda) W_{\lambda}(\lambda, T) \mathrm{d} \lambda
$$

$\varepsilon(\lambda, T)$ is the spectral emissivity of the sample within the collected solid angle, $F(\lambda)$ is the transmittance of the IR optics, $R^{*}(\lambda)$ the relative spectral responsivity of the detector, and $W_{\lambda}(\lambda, T)$ Planck's blackbody radiation. The constant factor $C$ includes the solid angle of the collected radiant flux, the emitting surface area, the maximum responsivity $R_{\max }$ of the detector, the amplification factor of the used electronic components etc. The radiometric signal $\delta M$, which is measured for the thermal wave response $\delta T$, is a small additional contribution to the signal $M(T)$. Thus it can be deduced from equ. (1) as partial derivative with respect to the temperature $T$. If the temperature variation of the effective emissivity is negligible in comparison to the temperature dependence of Planck's blackbody radiation, the photothermal signal measured for thermal waves can be approximated by

$$
\delta M(f, T)=C \varepsilon_{\text {eff }}\left(T, \lambda_{1}, \lambda_{2}\right) \int_{\lambda_{1}}^{\lambda_{2}} F(\lambda) R^{*}(\lambda) \frac{\partial W_{\lambda}(\lambda, T)}{\partial T} \mathrm{~d} \lambda \cdot \delta T(f)
$$

Here $\delta T(f)$ is the thermal wave of the solid, which depends on the heating modulation frequency $f$. When thermal waves for the example of an opaque two-layer solid are considered, the complex frequency-dependent solution at the sample surface is given by

$$
\delta T_{s}\left(x_{s}=0, f, t\right)=\frac{\eta_{s} I_{o}}{2 e_{s} \sqrt{2 \pi f}} \frac{\left[1+R_{s b} \exp \left(-2 \sigma_{s} \ell_{s}\right)\right]}{\left[1-R_{s b} \exp \left(-2 \sigma_{s} \ell_{s}\right)\right]} \exp [i(2 \pi f t-\pi / 4)]
$$

and when thermal waves for a homogeneous opaque solid are measured, the solution is

$$
\delta T_{s}\left(x_{s}=0, f, t\right)=\frac{\eta_{s} I_{o}}{2 e_{s} \sqrt{2 \pi f}} \exp [i(2 \pi f t-\pi / 4)]
$$

In equ. (4) and (4a), $\eta_{S}$ is the not reflected fraction of the incident intensity $I_{0}, e_{S}$ is the effusivity of the surface layer, and $\ell_{s}$ its thickness. The quantity

$$
\sigma_{s}=(1+i) \sqrt{\pi f / \alpha_{s}}
$$

with $i$ the imaginary unit and $\alpha_{\mathrm{s}}$ the thermal diffusivity of the surface layer is complex and contributes to the phase shift of the thermal wave. The quantity

$$
R_{s b}=\left(e_{s} / e_{b}-1\right) /\left(e_{s} / e_{b}+1\right)
$$

can be considered as a thermal reflection coefficient at the transition of the surface layer to the substrate of effusivity $e_{\mathrm{b}}$.

For the quantitative interpretation, the measured signals, both amplitudes and phases, are normalized by using reference signals measured under equal conditions of focussing for a homogeneous, opaque and comparatively thick sample of glassy carbon (Sigradur) of well known thermal and optical properties. If we assume that the stationary temperatures of the sample and reference are equal, the integral which describes the effects of the IR components on the measured signal, namely the spectral transmittance and responsivity, is eliminated by the normalization procedure and we obtain

$$
\frac{\delta M_{r}(T, f)}{\delta M_{s}(T, f)}=\frac{\varepsilon_{e f f, r}\left(T, \lambda_{1}, \lambda_{2}\right) \eta_{r}}{\varepsilon_{e f f, s}\left(T, \lambda_{1}, \lambda_{2}\right) \eta_{s}} \frac{e_{s}}{e_{r}} \frac{\left[1-R_{s b} \exp \left(-2 \sigma_{s} \ell_{s}\right)\right]}{\left[1+R_{s b} \exp \left(-2 \sigma_{s} \ell_{s}\right)\right]}
$$


If additionally the radiometric signal corresponding to the stationary sample temperature $T$ is measured for the coated sample as well as for the reference body, the ratio of the effective emissivities can also be eliminated and we obtain

$$
S_{n}^{-1}\left(f^{-1 / 2}\right)=\frac{\delta M_{r}(T, f)}{\delta M_{s}(T, f)} \frac{M_{s}(T, f)}{M_{r}(T, f)}=\frac{\eta_{r}}{\eta_{s}} \frac{e_{s}\left[1-R_{s b} \exp \left(-2 \sigma_{s} \ell_{s}\right)\right]}{e_{r}\left[1+R_{s b} \exp \left(-2 \sigma_{s} \ell_{s}\right)\right]}
$$

A signal interpretation according to equ. (8) relies on the assumption that the radiometric signal is generated at the surface of the two-layer solid. Radiative contributions to the measured signals $\delta M(T, f)$ from below the surface, which in principle are possible in amorphous diamondlike carbon layers, are neglected here, whereas radiative as well as conductive contributions to the heat transport inside the solid are described by the thermal diffusivity $\alpha$ and the effusivity $e$.

\section{Experimental results}

In figure 2 the photothermal signal amplitudes are presented which have been measured for four coatings of $4 \mu \mathrm{m}$ thickness on steel substrates. One of them consists of diamond-like carbon (dark gray), the other three additionally contain metallic additives contributing to a silvery, light gray, and golden colour, respectively. The signals measured for the dark gray sample with the DLC film are about 10 times above the others, mainly due to a higher photothermal efficiency $\eta_{\mathrm{s}}$, corresponding to a smaller reflectivity in the visible. The measured signal amplitudes of the dark gray sample already show a variation in the frequency dependence according to equation (4), as expected for layered samples. The signals of the other samples, the coatings of which contain metallic additives, show a constant frequency dependence $1 / \sqrt{f}$, corresponding to equation (4a) and expected for a homogeneous solid. This is probably due to the fact that the difference of the effusivities between the coatings and the substrate is too small, giving a thermal reflection coefficient of about $R_{\mathrm{sb}} \approx 0$.

In figure 3 the inversely normalized amplitudes of the DLC-coated sample are plotted versus the inverse of the square root of the modulation frequency. This representation of the amplitudes gives information about the distribution of the effusivity with increasing penetration depth. The measured data are compared in a first approximation with the two-layer model with an opaque surface-layer (equ.7). The deviations between measurement and theoretical model in the limit of high frequencies corresponding to a small penetration depth can be removed, when a slightly transparent first layer is assumed for the measured smooth surface [4]. This is shown in figure 4, where the normalized phases are represented versus the square root of the modulation frequency and where for the higher modulation frequencies a perfect agreement between measured data and theoretical interpretation is achieved. The deviations between measured data and theoretical model at low frequencies can additionally be removed if we admit a continuous transition between coating and substrate.

The data obtained for the DLC coating by the approximation of the phases are $e_{\mathrm{s}} / \mathrm{e}_{\mathrm{b}}=$ 0.36 for the ratio of the effusivities of the coating and the substrate, $\tau_{\mathrm{s}}=\ell_{\mathrm{s}}{ }^{2} / \alpha_{\mathrm{s}}=12.25 \mu \mathrm{s}$ for the thermal diffusion time of the coating, and $\beta_{\mathrm{s}}=1.9 .10^{6} \mathrm{~m}^{-1}$ for the absorption constant in the visible. The thermophysical parameters are determined at intermediate modulation frequencies, independently of the optical absorption constant, whereas this latter parameter is determined in the range of the high modulation frequencies. Based on the known thickness of the coating, $\ell_{\mathrm{s}}=4 \mu \mathrm{m}$, the thermal diffusivity of the coating can be calculated from the thermal diffusion time to be $\alpha_{\mathrm{s}}=1.31 \cdot 10^{-6} \mathrm{~m}^{2} \mathrm{~s}^{-1}$.

The effusivity value of the coating, which is low in comparison to that of the steel substrate, $e_{\mathrm{s}}<e_{\mathrm{b}}$, contributes to a reduced thermal transport between the fluid lubricant and the metal component. In transient friction processes, this may lead to comparatively higher temperature differences between the lubricant, the surface temperature and the temperature 
of the metal component. This effect however is limited in time due to the limited thickness of the coating, corresponding to a limited thermal diffusion time.

In figure 5, the inversely normalized amplitudes measured for a metallized diamond-like carbon film $(\mathrm{W}-\mathrm{C}: \mathrm{H})$ of $2.5 \mu \mathrm{m}$ thickness with metallic bond layers of $0.2 \mu \mathrm{m}$ tungsten and 0.3 $\mu \mathrm{m}$ of chromium on a steel substrate are presented, namely for the virgin sample and for sample positions with friction marks. For the virgin sample and the sample positions with friction marks, good agreement between the measured data and the theoretical approximation is achieved, both for high and low modulation frequencies, when a two-layer model with a slightly translucent surface layer is used for the interpretation. This is demonstrated by the normalized phases in figure 6 , where the negative phase values in the high frequency range serve to determine the absorption coefficient.

The data obtained for the virgin sample by the approximation of the phases are $e_{s} / e_{b}=$ 0.21 for the ratio of the effusivities of the coating and the substrate, $\tau_{s}=\ell_{s}{ }^{2} / \alpha_{s}=5.9 \mu$ for the thermal diffusion time of the coating, and $\beta_{\mathrm{s}}=3.0 .10^{6} \mathrm{~m}^{-1}$ for the absorption constant in the visible, which means that part of the incident light is absorbed at the coating-substrate interface.

If we compare the samples with and without friction marks, first qualitative statements based on the amplitudes in figure 5 are that the thermal diffusion time $\tau_{\mathrm{s}}=\ell_{\mathrm{s}}{ }^{2} / \alpha_{\mathrm{s}}$ of the surface layer of the samples exposed to friction is larger than that of the virgin sample and that the ratio of the effusivity values of the surface layer to that of the substrate, $e_{s} / e_{b}$, of the friction-exposed sample is smaller than the corresponding ratio for the virgin sample. This statement is confirmed by the normalized phases (figure 6). When we interprete the normalized phases obtained for the samples with and without friction marks and assume that a roughness- or stress-increased thickness of the layer of reduced thermal transport parameters can be excluded, the increased thermal diffusion time $\tau_{\mathrm{s}}=\ell_{\mathrm{s}}{ }^{2} / \alpha_{\mathrm{s}}$ measured after friction exposure is due to a reduced value for the thermal diffusivity. On the basis of the resulting values for the effusivity and thermal diffusivity obtained from the approximation based on the two-layer model with a slightly translucent first layer, the thermal conductivity $k_{\mathrm{s}}$ $=e_{\mathrm{s}} \alpha_{\mathrm{s}}^{1 / 2}$ and the specific heat $(\rho c)_{\mathrm{s}}=e_{\mathrm{s}} / \alpha_{\mathrm{s}}^{1 / 2}$ have been evaluated for the surface layers.

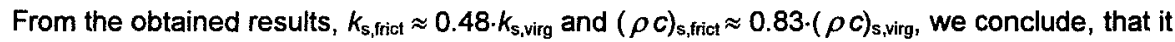
mainly is the thermal conductivity $k_{\mathrm{s} \text {,frict }}$ of the protective layer which is reduced owing to friction wear and induced stresses, and not the heat capacity $(\rho c)_{s, \text { frict. }}$. The reduced thermal conductivity, the reduced effusivity and the increased thermal diffusion time, measured for the coating samples with friction marks, indicate that the heat transfer from the lubricant to the metallic components diminishes with the progress of friction wear, and that thus the temperature of the lubricant can increase.

\section{Conclusions}

In thermal depth profiling of amorphous DLC films with thicknesses of 2.5 to $4 \mu \mathrm{m}$, it was possible to distinguish between the thermal parameters at intermediate modulation frequencies and the optical absorption coefficient at high modulation frequencies. This was achieved by using IR detection of thermal waves with an interval of the modulation frequencies extended up to $80 \mathrm{kHz}$.

Based on a two-layer model with a slightly translucent first layer, it was thus possible to determine the thermal parameters, relevant for the characterization of relatively dark a-C:H films used in tribological systems. By a comparison of samples with and without friction marks, it was additionally possible to identify and quantify the effects of friction wear and induced stresses. It has been found that it is mainly the thermal conductivity of the protective 


\section{http://dx.doi.org/10.21611/qirt.1996.033}

film, which is reduced owing to the exposure to friction.

For DLC films with a higher fraction of metalic additives, we failed so far in measuring reliable thermal depth profiles. This may be due to two facts: (i) The thermal contrast between coating and substrate is too small, and (ii) the reflectivity of such samples is higher, resulting in a too low power deposited by the laser heating beam at the high modulation frequencies, required to resolve such thin layers.

\section{REFERENCES}

[1] HOLLAND (J.), LINNENBRÜGGER (A.), TYCHSEN (M.). - Fundamentals of friction. Tribologie + Schmierungstechnik 38/6, 1991, p. 351.

[2] REBSCH (H.), JOST (M.), DEBUS (K.), BOSSE (H.), FLEISCHER (G.). - Optimierung des Gleitverhaltens zwischen metallischen Kufenwerkstoffen und Eis. Tribologie + Schmierungstechnik 38/6, 1991, p. 346.

[3] BEIN (B.K.), GU (J.H.), MENSING (A.), SOMMER (T.), WUNDERLICH (B.), PELZL (J.), SEIDEL (U.). - Modulated IR Radiometry of Rough Surfaces at High Temperatures. Balageas (D.), Busse (G.), Carlomagno (G.M.) eds., Quantitative InfraRedThermography QIRT 92. Paris, Editions Européennes Thermique et Industrie, p. 393-398, 1992.

[4] KALUS (G.). - Untersuchung thermischer Eigenschaften und Dicken von Plasmabeschichtungen mit Infrarot-Radiometrie. Diploma Thesis, Ruhr-Universität Bochum, 1995.

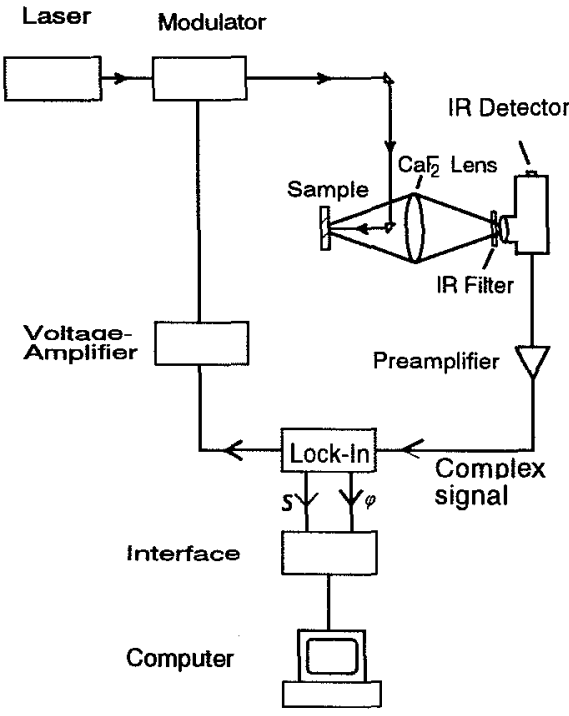

Fig. 1. Schematic of the system for the IR detection of thermal waves.

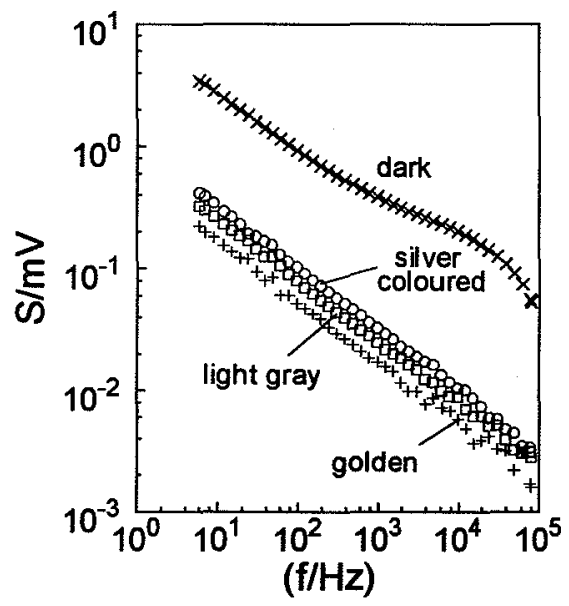

Fig. 2. Photothermal signal amplitudes measured for DLC coatings of $4 \mu \mathrm{m}$ thickness on steel. The dark gray coating consists of $\mathrm{a}-\mathrm{C}: \mathrm{H}$, the others contain metallic additives. 


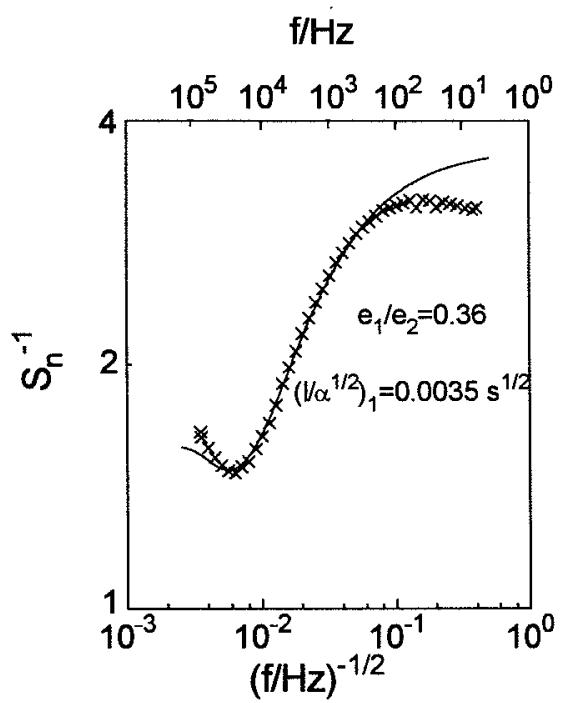

Fig. 3. Inversely normalized amplitudes for a $4 \mu \mathrm{m}$ thick a-C:H coating on steel, versus the inverse of the square root of modulation frequency. - In the presented form, measured data give an impression of the effusivity profile.

$\mathrm{f} / \mathrm{Hz}$

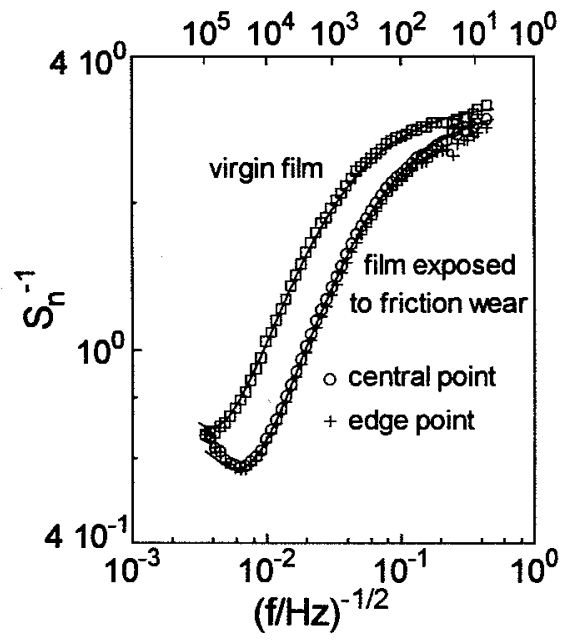

Fig. 5. Inversely normalized amplitudes for $2.5 \mu \mathrm{m}$ thick $\mathrm{W}-\mathrm{C}: \mathrm{H}$ coatings with a metallic bond layer $(0.2 \mu \mathrm{m} \mathrm{W}$ and $0.3 \mu \mathrm{m} \mathrm{Cr}$ ) on steel. - Measurements for the virgin sample are compared to samples with friction marks.

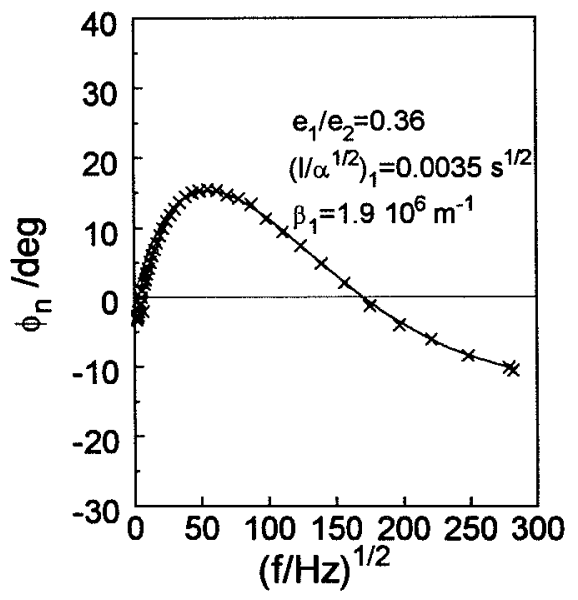

Fig. 4. Normalized phases for an a-C:H coating $(4 \mu \mathrm{m})$ on steel, in comparison to a theoretical approach based on a twolayer model with a slightly translucent first layer.

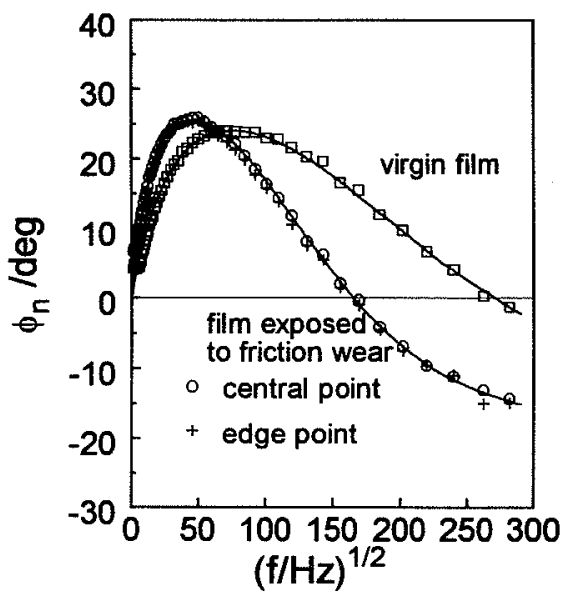

Fig. 6. Normalized phases for W-C:H coatings (virgin sample and samples with friction marks), in comparison to a theoretical approach based on a two-layer model with a slightly translucent first layer. 